\title{
IBD background rejection and tagging at the Double Chooz experiment
}

T. Brugiere, A. Hourlier, C. Jollet, K. Kale Sayi, M. Lopez Castano, A. Meregaglia, C. Palomares, V. Sibille, R. Sharankova, Guang Yang ${ }^{\dagger *}$

${ }^{\dagger} E$-mail: gyangenngroup.physics. sunysb. edu

The Double Chooz experiment is a reactor neutrino disappearance experiment located at the Chooz nuclear power plant in France. The primary goal of the Double Chooz experiment is to precisely measure the neutrino mixing angle $\theta_{13}$, a neutrino oscillation parameter. In this paper, the main background sources and rejections are shown, as well the measurements of the remaining background rates and shapes.

38th International Conference on High Energy Physics 3-10 August 2016

Chicago, USA

* Speaker. 


\section{Introduction}

Double Chooz was built to measure the neutrino mixing angle $\theta_{13}$. The experiment consists of two identical liquid scintillator detectors and measures the electron-antineutrino flux of the two nuclear reactors. The $1 \mathrm{~km}$ distant far detector (FD) started operation in 2011. The $400 \mathrm{~m}$ distant near detector (ND) started operation at the end of 2014. In this paper, we include the data set till the end of 2015. The reactor neutrinos are detected by the signature of an inverse beta decay (IBD). Inverse beta decay provides a unique prompt-delayed coincident signal to identify the electron antineutrinos from the reactors. The high correlation between the near and the far detector can significantly suppress the systematics for oscillation measurements. The neutrino energy spectrum is extracted from the spectrum of the IBD-produced positrons. The IBD-produced neutrons can be captured by Gadolinium (nGd) or Hydrogen $(\mathrm{nH})$, which provides two independent data samples. Both samples allow the utilisation of the neutrino rate and energy spectral shape information in a combined fit. The parameter $\theta_{13}$ is extracted by a simultaneous fit to the data observed in the two detectors. Therefore, backgrounds(BGs) are important for the $\theta_{13}$ measurement as they may affect the IBD prompt spectra in both detectors.

\section{Background in DC}

The DC detectors have an outer veto and an inner veto detection systems in order to tag the background events. The outer veto consists of hundrads of scintillator strips on the top of the cylindrical detector. The cylindrical inner veto surrounds the inner detector and is filled with liquid scintillator and equipped with 78 8" PMTs. More details can be found in [1]. The overburden of the near detector is smaller than that of the far detector so we expect more cosmogenic backgrounds in the near detector. There are three main kinds of background existing in DC. They are described as following.

The first one is accidental background, which caused by two "piled-up" low energy ambient radioactive events. Since the prompt and delayed events of the accidental background are uncorrelated while those of IBD are correlated, we use the time and space differences between the prompt and delayed signals as well as the delayed energy information to separate the signals and backgrounds. A ROOT-based artificial neural network (ANN) has been developed for this separation. Fig. 1 shows the ANN output distributions for signal MC, accidental BG data, total data and BG subtracted data. A clear separation between the signal and BG can be seen. The remaining accidental background rate and shape can be measured using an off-time window technique. The coincidence time window after the prompt event has been slided to $1 \mathrm{~s}$ away and 2,000 such windows for each event in the $\mathrm{nGd}$ analysis are used in order to extract the accidental BG rate and shape.

The second kind of background is fast neutrons/stopping muons. Due to similar origins and features, they are combined. The stopping muon background is generated when the cosmogenic muons pass through the detector chimney area and decay to michel electrons. The stopping muons are almost fully rejected by employing a combination of cuts, including a point-like likelihood cut for the delayed events and a chimney event pulse shape likelihood cut. More details can be found in [1]. Left panel in fig. 2 shows the rejected stopping muon time difference distribution. The 


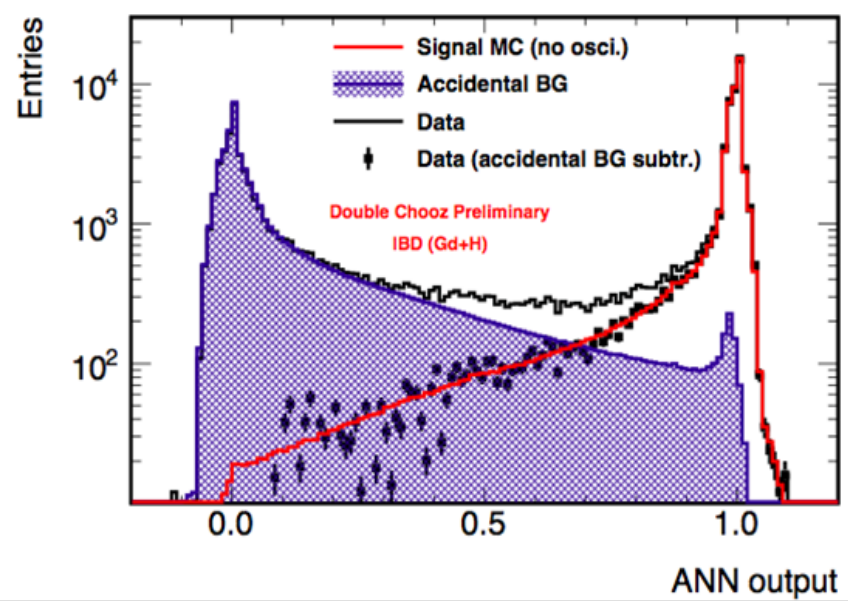

Figure 1: ANN output distributions for signal MC, accidental BG data, total data and BG subtracted data.

decay time for these rejected events is consistent with the muon life time. The fast neutrons are generated due to the high energy cosmogenic muon spallations in the surrounding rocks. When the fast neutrons go through the inner detector, the proton recoil and neutron capture can mimic the signal coincidence. Since the fast neutrons have high energy, the prompt proton recoil events extend to a very high energy. Differences in the prompt event pulse shape between fast neutrons and IBD, due to multiple proton recoils in the FN signal, can be used to discriminate between them. The shape of the remaining fast neutrons is obtained based on the inner veto tagged events and the rate based on the event rate in the high energy region (20-100 MeV) after the BG rejections. Right panel in fig. 2 shows the background components before the oscillation analysis. High energy region is dominated by the fast neutron events.
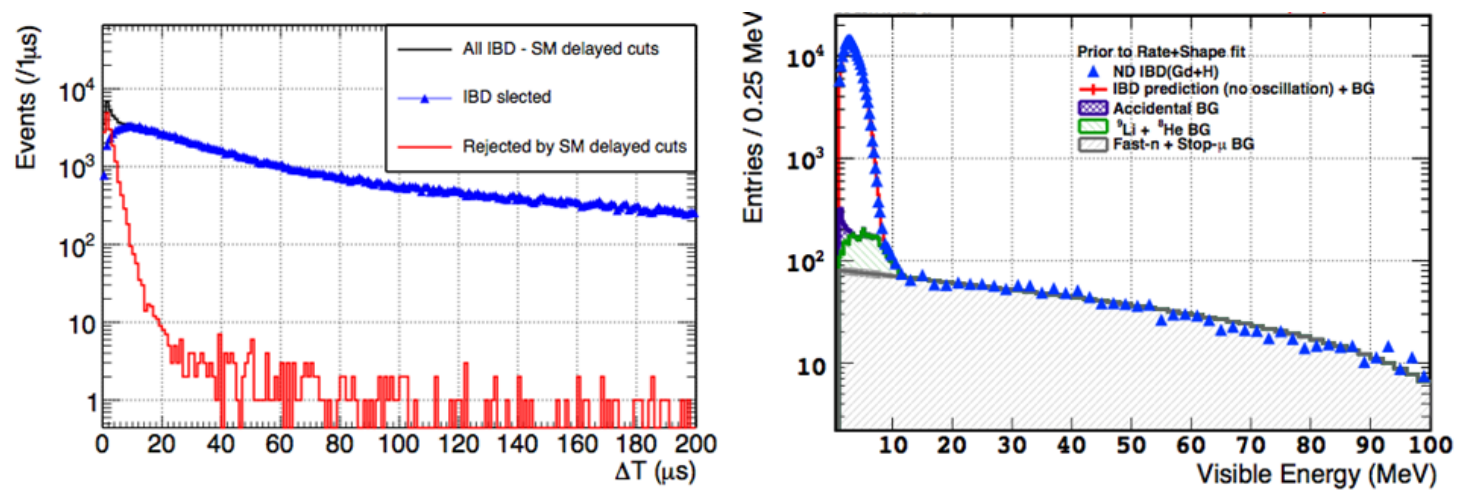

Figure 2: Left: Stopping muon event time difference distribution. Right: Background components in the prompt energy spectrum before the oscillation analysis.

The last kind of background is ${ }^{9} \mathrm{Li}$, a $\beta-\mathrm{n}$ generator. It is generated from Carbon receiving energy from the cosmogenic muons. Another dedicated ICHEP proceeding addresses this background. Also, more details can be found in ref. [2]. The mechanisms of causing the three kinds of background are shown in fig. 3. 


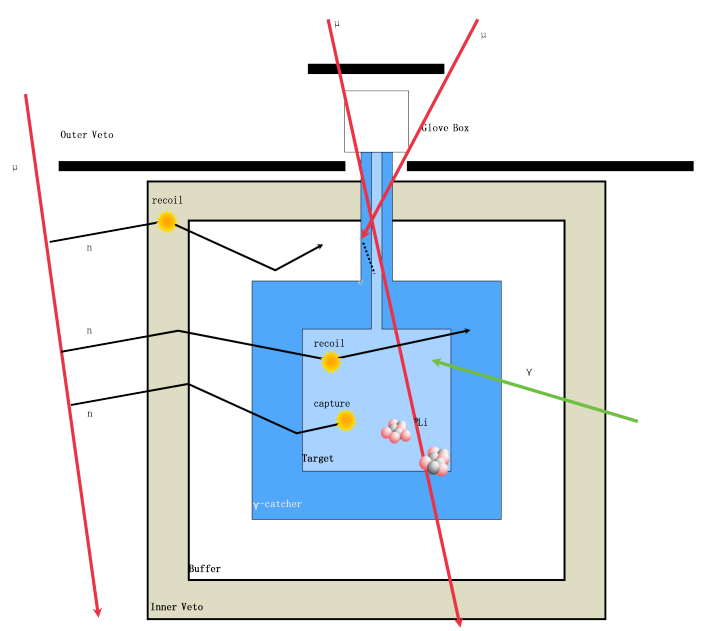

Figure 3: Mechanisms of generating the backgrounds. See the text for details.

\section{Reactor-off measurement}

The reactor power company, Électricité de France, switched off both reactors in order to do the maintainance for 7.6 days. This period is used to measure the backgrounds and this measurement provides direct constraint on the background rates. More details can be found in ref. [3].

\section{Summary}

The background rates for the nGd channel analysis for FDI (data taken from 2011-2014), FDII (data taken from 2015) and ND ( data taken from 2015) are summarized in tab. 1. The ratios of signal to background are also included. These numbers are input to the oscillation analysis and the background rates are varied in the oscillation analysis with $\theta_{13}$. More details on the oscillation analysis can be found in the ICHEP DC oscillation analysis proceeding.

\begin{tabular}{cccc}
\hline \hline Detector & FDI & FDII & ND \\
\hline Accidentals (per day) & $0.069 \pm 0.002$ & $0.118 \pm 0.006$ & $0.303 \pm 0.007$ \\
Fast neutrons (per day) & $0.43 \pm 0.03$ & $0.51 \pm 0.04$ & $4.00 \pm 0.15$ \\
Li (per day) & $0.87 \pm 0.42$ & $0.87 \pm 0.42$ & $4.67 \pm 1.42$ \\
Reactor off (per day) & $0.76 \pm 0.38$ & NA & NA \\
Signal to BG ratio & 23.80 & 27.00 & 31.50 \\
\hline
\end{tabular}

Table 1: Summary of the background rates in DC.

\section{References}

[1] Double Chooz collaboration, JHEP 10 (2014) 086 [Erratum ibid. 02 (2015) 074].

[2] Double Chooz collaboration, Phys. Rev. C 93, 054608 (2016).

[3] Double Chooz collaboration, Phys. Rev. D 87, 011102 (2013). 